\title{
Time Resolved IR and X-Ray Simultaneous Spectroscopy: New Opportunities for the Analysis of Fast Chemical-Physical Phenomena in Materials Science
}

\author{
A. Marcelli ${ }^{a}$, D. $\operatorname{Hampai}^{a}, \mathrm{Wei}_{\mathrm{XU}}{ }^{a, b}, \mathrm{~L}$ Malfatti $^{c}$ \\ AND P. INNOCENZI ${ }^{c}$ \\ ${ }^{a}$ Laboratori Nazionali di Frascati - INFN, Via E. Fermi 40, 00044 Frascati, Rome, Italy \\ ${ }^{b}$ Institute of High Energy Physics, Chinese Academy of Science, Beijing 100049, China \\ ${ }^{c}$ Laboratorio di Scienza dei Materiali e Nanotecnologie, Università di Sassari \\ D.A.P., CR-INSTM, Palazzo Pou Salit, Piazza Duomo 6, 07041 Alghero, Sassari, Italy
}

\begin{abstract}
New powerful sources and advanced analytical techniques have been considered in the last decade to face up the continuously increasing scientific demands, in particular, in materials science. As an example, nanoscience and nanotechnology researches are characterized by ultimate spatial resolution, fast and ultrafast time-resolved analysis, but the complexity of the investigated phenomena requires new analytical capabilities and new experimental techniques were introduced in the research arena. The availability all over the world of brilliant synchrotron radiation sources offers incredible opportunities. Many challenging experiments were made possible by these sources and understanding of many complex dynamical problems was obtained. Nevertheless, a strong demand of new analytical approaches, mainly based on concurrent and possibly simultaneous time-resolved experimental techniques, is emerging. Pioneering time resolved experiments combining X-ray and infrared radiation with a conventional source were performed more than a decade ago. Nowadays, many beamlines at third generation synchrotron radiation facilities are equipped with conventional sources to allow complementary techniques and the strategy of a concurrent analysis is mandatory in the investigation of many phenomena in frontier multidisciplinary researches. Moreover, new opportunities will be available by means of concurrent spectroscopic experiments investigating complex phenomena on a short timescale, from the sub-second to the microsecond time domain. We will present and discuss researches where the combination of IR and X-ray simultaneous experiments may return unique information on complex dynamical processes and phase transitions occurring in materials science. Finally, we will briefly describe the conceptual layout of a synchrotron radiation beamline to perform concurrent IR and X-ray experiments.
\end{abstract}

PACS numbers: 07.85.Qe, 61.05.cj, 82.20.-w, 78.47.-p

\section{Introduction}

Nowadays, the availability all over the world of modern storage rings optimized for the synchrotron radiation (SR) emission in a wide energy range offers to scientists incredible opportunities in term of spatial and time resolution and allow performing many different experiments providing answers to complex problems. Triggered by nanoscience and nanotechnology demands, techniques characterized by ultimate spatial resolution, fast and ultrafast time-resolution are now available at many SR facilities to many users. Nevertheless, the complexity of the investigated phenomena does not require only intense and/or brilliant sources and simultaneous analytical capabilities are sometimes mandatory.

In particular, in order to fulfill the continuously increasing scientific demands, we experience a clear lack of experimental methods based on concurrent and possibly simultaneous time-resolved capabilities. The advantages of X-ray investigations are well recognized, in particular, in materials science for structural analysis using scattering or spectroscopic experiments although the most demanding X-ray experiments can be performed only using SR sources. However, the most diffused research technique and also widely used in the industry to characterize materials and processes is the Fourier transform infrared spectroscopy (FTIR), a well established method based on conventional sources. It is used to characterize all molecular systems and it is capable to follow fast chemical reaction kinetics and chemical changes occurring simultaneously [1]. It is then straightforward to image that the combination of the X-ray atomic and structural analysis with the IR molecular analysis will offer high rewards to materials science improving the understanding of complex materials and phenomena.

In this contribution we will discuss why and how it is possible combining X-ray with IR synchrotron radiation beams and in particular, what kind of simultaneous $\mathrm{X}$-ray and IR spectroscopy experiments can be investigated with this hybrid method. 
Pioneering time resolved experiments combining X-ray and infrared radiation were performed at Daresbury more than a decade ago combining time-resolved FTIR with small angle X-ray scattering to investigate an organic polymer structure. The experiment provided the first evidence of a time correlation between reaction kinetics and structure developments [2]. Although the concept of combined techniques were already accepted and successful experiments were previously reported, the experiment performed by Bras et al. exploited the significant advantage of using SR for small-angle X-ray scattering (SAXS) experiments for polymer processing investigations, e.g., the high X-ray intensity allowed time-resolved X-ray scattering experiments to be performed with a time resolution of several minutes comparable to the time processes occurring in polymers (see Fig. 1).

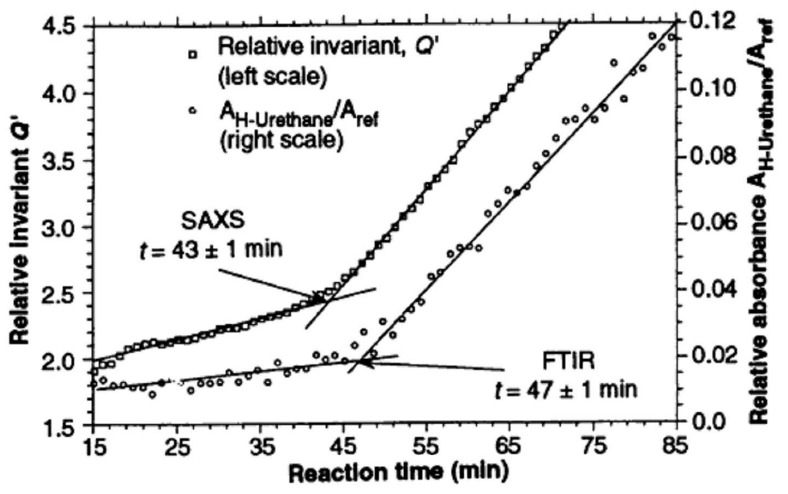

Fig. 1. The SAXS relative invariant $Q^{\prime}$ (left) and the normalized FTIR absorbance (right) plotted vs. time. Taking into account the perfect time correlation of the two techniques and the observed behaviors during this polymer process authors demonstrated that the microphase separation start before hydrogen bonding (see Ref. [2] for more details).

The study provided a useful insight into the morphological changes that take place in these materials during a phase transition. However, in this experiment, both the dimensions of the sample and the timescale of the process did not require the use of a brilliant IR synchrotron radiation source and experiments were performed coupling an interferometer equipped with a Globar source and an ATR crystal to monitor the sample response at IR wavelengths. However, polymers are only one class of materials and many other materials and processes where a complex combination of both chemical and physical events occur, may be investigated by time-resolved techniques. Concurrent experiments on complex physical-chemical phenomena may obtain unique and accurate information about a process, leading to a deeper understanding of the simultaneous processes occurring on different time or energy scales. Moreover, when time-resolved experiments are performed simultaneously, a wealth of information can be derived from the same sample, and artifacts always present combining two independent (and time delayed) experimental data sets, e.g., due to temperature or thermal history differences or time mismatch between the two experiments, can be neglected. New opportunities will be open by concurrent spectroscopic experiments investigating new materials and/or complex phenomena on a short timescale. In the next sections we will present and discuss a few examples that may explain why the combination of infrared and X-ray simultaneous experiments may be usefully applied to understand dynamical processes and phase transitions. In particular, we will discuss applications in self-assembly phenomena [1] where the combination of two simultaneous spectroscopic analyses such as X-ray and IR spectroscopy may return complementary information on different aspects of the process, e.g., structural changes and chemical processes occurring during the ordering phenomena. Moreover, triggered by the booming of many research areas such as nanotechnologies and bio-engineering, in addition to a fast time-resolved analysis a demand of new advanced analytical techniques characterized by a high spatial resolution (micro- to nanoscale) and simultaneous analytical capabilities to characterize the complex structures and the different interplaying interactions is emerging. The simultaneous combination of techniques will certainly have a significant impact on the understanding of phase transitions, an improved knowledge of materials and of their synthesis processes, and in the future will probably lead to the growth of new and better materials. As a consequence, in the last section we will briefly describe the conceptual layout of a synchrotron radiation beamline designed to perform concurrent infrared and X-ray experiments. The scientific opportunities discussed in this manuscript actually are only a limited set among the many experimental opportunities, but may well explain why nowadays at many SR beamlines conventional source instrumentations are available, allowing complementary analysis, witnessing how the combination of experimental techniques is a clear trend at third generation SR facilities $[3,4]$.

\section{Scientific opportunities}

As outlined in the introduction and by Newton et al. in Ref. [5] many fundamental chemical processes and in particular catalysis processes involve a complex interplay of fundamental physical and chemical phenomena over a wide range of parameters such as the space, the energy, and the time. The opportunity to investigate in situ phase transitions with concurrent spectroscopies is a really new powerful method and simultaneous experiments that combine at least two complementary techniques such as X-ray and FTIR analytical techniques may offer the possibility to obtain accurate quantitative relationships e.g., vs. time or other relevant parameters of a process such as relative molecular contents.

In the last decade several experiments combining different methods have been implemented probing simultaneously structure and dynamical parameters in particular using techniques sensitive to long and short-range 
order of atoms and molecules, such as X-ray diffraction (XRD) and X-ray absorption (XAS) [6, 7]. Extended Xray absorption fine structure (EXAFS) experiments investigating processes in combination with UV-vis and/or IR [8-13] have been applied to investigate in situ catalytic processes at both ambient and high pressures and for both gas-solid and liquid-solid systems. However, some concepts have to be clarified regarding the best combination of techniques and, in particular, the evaluation of cost/benefit and the real advantages of the use of both IR and X-ray synchrotron radiation sources for simultaneous time-resolved experiments has to be performed and discussed. X-ray diffraction and absorption spectroscopy are techniques widely used to investigate crystal and local structure, respectively, and the different degree of long and short-range order, while vibrational spectroscopy techniques may probe matter on length scale comparable to the wavelength used (few micrometers) actually intermediate between the sensitivity of XRD and $\mathrm{XAS}$ and it is sensitive to the molecular components. Actually, XAS spectroscopy is a reliable probe of the electronic configuration, e.g., the valence state and it is sensitive to the local and partial empty density of states [14]. As recently illustrated by Phillips and Quake [15] thermal, chemical, mechanical and electrostatic energies scale with the size of an associated object and the theoretical behavior is very remarkable because indicates the occurrence of the confluence of energies as the characteristic size approaches that of biological macromolecules as described in Fig. 2 in Ref. [15], e.g., ranging from a few $\mathrm{nm}$ to hundreds of $\mathrm{nm}$. The occurrence of a convergence at the molecular scale is really stimulating and addresses the existence of an effective interplay between thermal effects such as diffusion or conformational changes and deterministic forces in many complex phenomena or systems, e.g., DNA molecules which are at the base of the living systems [15].

In general, combining a time-resolved structural and electronic analysis with a time-resolved molecular analysis may allow:

- identify and characterize time-dependent physical-chemical phenomena;

- provide time-resolved information on driving mechanisms of different phenomena.

The synergy between X-ray and IR methodologies is evident and addresses a lot of advantages.

The first combined simultaneous XAS/IR experiments were also performed at Daresbury at the station 9.2 of the SRS storage ring. Using Globar and SR sources, combined Fourier-transform IR and X-ray absorption spectroscopy studies were performed on $\mathrm{NiBr}_{2}$ isolated in a nitrogen matrix and later on matrix isolated species in the vapor phase $[16,17]$. Experiments were performed with a time resolution of a few s on samples of $c a .2-3 \mathrm{~mm}$ of size, a dimension for which we do not take advantage of the intrinsic brilliance of the SR emission in the IR domain [18]. However, reducing the area of the investigated sample, thanks to the brilliance of all SR sources, significant gain can be obtained and experiments impossible to be performed with a conventional source can be realized. At present, time-resolved phenomena that could take benefit by the availability of a hybrid simultaneous modality are:

- phase transitions and self-assembly processes,

- order/disorder phenomena,

- nanosystems or processes affected by a low dimensionality,

- biosystems,

- surfaces and interfaces processes,

- processes associated to biointeractions,

- unfolding processes of (metallo-)proteins,

- radiation damage or radiation resistance phenomena, etc.,

and in general, all experiments looking at chemical and structural modifications triggered by external parameters such as temperature, $\mathrm{pH}$, concentrations, mole, $E$, $B$, humidity, dose, etc. could be monitored with a dedicated setup on a SR facility performing experiments with a high time resolution on small samples. As showed in Ref. [1], IR simultaneous experiments make possible a semi-quantitative data analysis that can be later correlated to structural data provided by SAXS.

Scrolling at the above list the formation of an ordered mesophase in films a process characterized by self-assembly of the supramolecular template induced by solvent evaporation is one of the most interesting process to be investigated and still poorly understood [19]. A combination of colloidal and sol-gel chemistry controls the organization of a mesophase so that only in situ experiments may allow monitoring self-assembly processes, whose knowledge gives information of many competing phenomena and synthetic parameters. Many techniques have been introduced and are applied to the investigation of the self-organization in situ. The most powerful and well established one is certainly the SAXS with intense synchrotron radiation sources that allows monitoring different stages of the mesophase organization during the evaporation-induced self-assembly phenomenon in both liquids and thin films. In particular, time resolved SAXS experiments are fundamental to recognize changes in a mesophase induced by changes in the deposition variables even after the solvent evaporation [20]. To this purpose and in the attempt to clarify the existing correlation between chemical processes and structural changes occurring during the deposition of films it has been recently demonstrated that the combination in situ of two time-resolved analytical approaches, i.e., the FTIR and the SAXS may allow the discrimination between rates of different process (e.g., evaporation rates of different 
solvents vs. water). Recently, measurements have been performed at the Austrian SAXS beamline of the ELETTRA electron storage ring at Trieste combining the SR $\mathrm{X}$-ray radiation of Elettra with a conventional IR source. In these experiments the simultaneous technique was applied to the study of self-assembling mesostructured films during dip-coating processes [1]. A mother solution containing ethanol (EtOH), tetraethylorthosilicate (TEOS) water, $\mathrm{HCl}$, and a templating solution were prepared to obtain a final precursor sol by a mixing of the two solutions. A silicon substrate was dip-coated in the precursor sol and SAXS measurements were performed with an incident wavelength of $1.54 \AA$ at the critical angle of the mesostructured silica film. The simultaneous FTIR measurements were performed using a BRUKER IRCube interferometer, a compact commercial mid-IR spectrometer equipped with a $\mathrm{Ge} / \mathrm{KBr}$ beamsplitter, and a Globar source. The setup for simultaneous acquisition of grazing incidence X-ray scattering patterns and IR spectra in transmission mode is showed in Fig. 2. With this

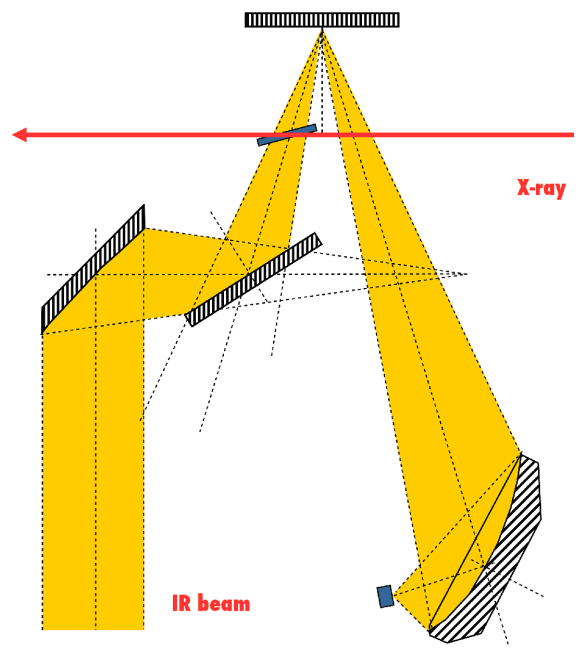

Fig. 2. Schematic drawing of the experimental setup used at Elettra for the simultaneous SAXS-FTIR time-resolved experiments. The SAXS analysis is performed in grazing incidence, and the infrared measure is performed in transmission mode. With this optical layout light coming from the bottom cross the sample and then is deflected and focus by an ellipsoid on a MCT detector.

optical layout the incident IR and X-ray beams probe the very same region of the sample. Data were collected with a liquid-nitrogen-cooled photoconductive detector and each spectrum was obtained at a resolution of $8 \mathrm{~cm}^{-1}$ every $2 \mathrm{~s}$. The time resolution is compatible with the timescale of the processes, e.g., of the order of seconds for both the self-organization and the chemical processes involved. Improved time resolution down to a few milliseconds should be in principle achieved with a similar experimental configuration in the rapid scan method using a different spectrometer and a fast detector. The real limitation of a Michelson interferometer is indeed the mirror speed so that faster acquisitions, extremely impor- tant to investigate faster processes, could be eventually obtained only by an IR spectrometer coupled to an array detector [21]. As it has been clearly demonstrated, simultaneous time-resolved FTIR and SAXS measurements in mesostructured silica films allow reconstruction of the kinetics of solvent and water evaporation, which can be correlated with structural data on mesophase ordering.

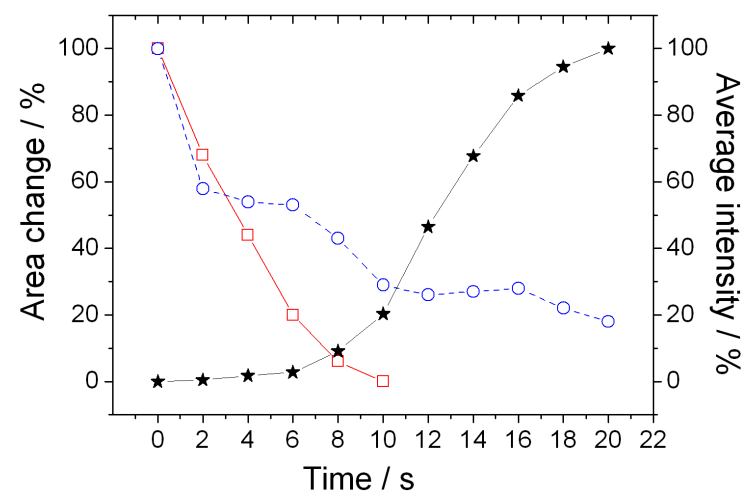

Fig. 3. Comparison of time-resolved phenomena with FTIR and SAXS. Curves report the changes in the area of the infrared bands at 1640 (water, squares) and 880 $\mathrm{cm}^{-1}$ (ethanol, circles) as a function of time after dipcoating. The evolution of the average intensity of the SAXS diffraction spot (black stars) as a function of time refers to the right scale. The lines are a guide for eyes.

As showed in Fig. 3 where FTIR (left part) and SAXS data (right part) were compared, in the first stage FTIR spectra are dominated by the signature of the evaporation of the ethanol and only after about $8 \mathrm{~s}$ this process is completed. From the simultaneous SAXS patterns it is also evident that within the first $8 \mathrm{~s}$ no mesophase order is observed. Looking at the trend of the intensity of the water vibrational modes a more complex evaporation process occurs for water. After a very fast initial evaporation an intermediate stage occurs between 2 and $10 \mathrm{~s}$ followed by a third very slow final evaporation phase, so that after $20 \mathrm{~s}$ while ethanol is completely evaporated still about $20 \%$ of the initial water is present. This work outlined for the first time a direct correlation from FTIR and SAXS spectra extracting information on the correlation of the water and solvent evaporations with the formation of an ordered mesophase within a film. In the right part of Fig. 3 is reported the behavior of the average intensity of SAXS diffraction spots vs. time that clearly indicates that the organization of the mesophase starts only after $10 \mathrm{~s}$, when silica walls are formed, i.e., just after the ethanol evaporation is completed and with a reduction of about $70 \%$ of the water content in the film. Although data show unambiguously that an ordering starts only after solvent evaporation, which is the critical step in all self-assembly processes, many questions regarding these complex phenomena remain open such as the instant of micelle formation or the role of the surfactant concentration. However, the advantage of a simultaneous IR and 
X-ray analysis, a completely not invasive approach that can be applied to thin films, cast drops, or solutions is evident. The process of evaporation during self-assembly is not a continuous process and different evaporation stages can be observed during which different phenomena occur. Figure 4 shows the 3D spectra obtained by a rapid scan FTIR acquisition of cast titania film in air. Infrared data clearly shows that whilst the evaporation of ethanol is a continuous process, water after the first initial evaporation stage is again increasing in the film due to adsorption from the environment and water produced during condensation reactions, both followed by a final evaporation stage. However, the water adsorption appears as a fast phenomenon associated to a second slow evaporation process [22]. Spectra associated to the first evaporation stage are accompanied by the presence of fringes due to changes of the thickness of the film that accompanied by a change of the dielectric constant suddenly disappear in correspondence of the transition from the liquid to the solid phase. The transition occurs on a fast timescale $(<\mathrm{s})$ and rapid scan methods with improved time resolution are required to monitor the vibrational behavior. In this case a simultaneous monitor with a time resolved X-ray spectroscopic method could clarify many local structural details of these complex phenomena and of the interactions with the atmosphere, all together fundamental information to understand how to guarantee the repeatability of technological processes based on solgel chemistry [23]. Among self-assembly processes, evaporation induced self-assembly (EISA) [24] represents a possible route to obtain self-organization. A remarkable example of EISA is self-patterning of complex structures by wrinkling in which evaporation of the solvent and condensation reactions are used to govern the formation of wrinkled structures. In some case, after the deposition, wrinkling can be used to produce patterns only if the film remains in the "liquid-like" state. UV illumination may be then used to induce wrinkling and finally to obtain organized oxide patterns [25]. The possibility to monitor this complex process with a simultaneous time-resolved X-ray spectroscopy may offer unique opportunities in the understanding of the chemistry and physics of materials processing during which evaporation of water, solvent and polycondensation reactions are involved at the same time. Several applications can be envisaged for a better understanding of the chemistry and physics of materials processing during which evaporation of water, solvent and polycondensation reactions are involved such as the deposition of sol-gel thin films via dip-coating and spin-coating. Time-resolved analytical methods are potentially important tools to follow the evolution of films deposited via a liquid phase because the overall process is a time-dependent phenomenon whose timescale depends on many parameters: the withdrawal speed during dip-coating, the type of solvent, the amount of solvent employed and/or the relative humidity in the deposition room. However, during a process the state of the sample may change and variations of the signal dur- ing a scan will affect the results and the following analysis. As a general rule, to minimize intensity changes during a rapid scan, the duration of the acquisition have to be at least one order of a magnitude shorter than the half-life of the dynamic event under investigation [20]. This requirement sets the limit to the feasibility of experiments with conventional sources (s) and of interferometric methods (ms).

Another class of experiments where time resolved simultaneous spectroscopy may offer unique opportunities is the metal-insulator transition (MIT). As it is well known from the last century, many transition-metal oxides such as $\mathrm{NiO}$ with a partially filled $d$-electron band are poor conductors and often insulators. Already in 1937 Peierls pointed out the importance of the electronelectron correlation and the role of a strong Coulomb repulsion between electrons regarding their insulating behavior [26]. Since then the research on strongly correlated electron systems continued and nowadays is still alive. Indeed, one of the challenging aspects of the condensed matter physics is the understanding of the emergence of metallic transport in correlated Mott insulators in which, for example, a temperature change or chemical doping induces anomalous conducting phases [27]. Many $d$-electron compounds have been investigated with the idea of systematically controlling the physical parameters tuning the metal-insulator transition. For example, by doping or heating we may induce anomalous conducting phases and a continuous theoretical and experimental effort is devoted to understand how partially filled bands could become insulators. When an insulator-to-metal transition is induced in a correlated insulator, the conducting electrons can have a radically different behavior if compared to free electrons in a conventional metal and different transport, optical and magnetic properties can be observed. Anomalous and unexpected phenomena such as high- $T_{\mathrm{c}}$ superconductivity and colossal magnetoresistance phenomena have been found in the region where the metallic state is retained close to the Mott insulator. The interest is not only the understanding of MIT but also the possibility to drive the system into the metallic state in a controllable way and take benefit from the strongly nonlinear responses observed in many materials in useful devices or for practical applications. Nonlinear responses offer a rich scenario of dynamic properties in particular in Mn perovskite compounds, good candidates for new optical or magnetic devices. In many Mn compounds we may observe a pressure-driven MIT. The volume compression has usually the effect of both increase the symmetry in the crystal structure and the overlap among the electronic clouds of ions and/or molecules leading to an increase in delocalization of the outer electrons. However, volume compressions may be also large and modify also the balance among microscopic interactions. Although complex and experimentally hard to perform, high pressure experiments are ideal tools to investigate complex systems where an interplay of different interactions occurs. While direct information about 
the electronic structure and structural properties can be obtained by X-ray absorption spectroscopic studies in a high pressure cell, high-pressure measurements on a manganite in the far IR domain become possible only thank to the availability of brilliant IR synchrotron sources [28]. $\mathrm{La}_{1-x} \mathrm{Ca}_{x} \mathrm{MnO}_{3}$ manganites in a wide doping range [29] may undergo to a transition from a high-temperature, paramagnetic, insulating phase to a ferromagnetic metallic phase. It is now widely accepted in manganites that the MIT originates from a competition between the ferromagnetic Mn ordering induced by itinerant holes through the double-exchange (DE) mechanism and an effective electron-phonon coupling due to the Jahn-Teller distortion of the $\mathrm{Mn}_{3} \mathrm{O}_{6}$ octahedra [30,31]. In Ref. [28] we find the first observation of a far-infrared (FIR) gap filling in a manganite, obtained by varying the pressure at room temperature using a diamond anvil cell (DAC) of $800 \mu \mathrm{m}$ culet equipped with IIA type diamonds and made possible by the intense synchrotron radiation emission in the far-IR domain. $\mathrm{La}_{1-x} \mathrm{Ca}_{x} \mathrm{MnO}_{3-y}$ samples with an oxygen deficiency showed a similar pressure dependence of the phonon spectrum, whereas only manganites with a metallic ground state at ambient pressure $\left(T_{\mathrm{MI}} \approx 200 \mathrm{~K}\right)$ show a clear trend towards a metallic character at room temperature vs. pressure. The possibility to combine these studies with an X-ray spectroscopy characterization can certainly improve the understanding of the transformation from an insulator to a metal not only in colossal magnetoresistive compounds but in many correlated electron systems that occurs through an intermediate pseudogap regime. We have to underline here that, in particular, in the case of experiments at high pressure using extremely small samples mounted inside a DAC, the possibility to perform concurrent experiments on the same sample and exactly within the same conditions may significantly minimize systematic errors, addressing also the problems of the irreproducibility of the experimental conditions inside DACs.

Another class of materials of great interest in which the combination of IR and X-ray spectroscopic analysis may return unique information is represented by clusters, e.g., assemblies of atoms whose corresponding bulk system is a metal or semiconductor. Actually, these weakly bound aggregates of condensed molecules typically exhibit different properties than their bulk counterparts. A lot of work in chemistry is devoted to the production of clusters with "special" properties to achieve catalysts with well defined properties.

Among clusters, fullerenes [32] are one of the most interesting classes. These molecules consisting of carbon, form a closed sphere structure built up out of hexagons and pentagons. Actually, according to the Euler theorem each fullerene consists out of 12 pentagons and a finite number of hexagons. Besides, for energetic reasons, every pentagon has to be surrounded by hexagons (isolated pentagon rule). The smallest sphere fulfilling these conditions is $\mathrm{C}_{60}$, also known as Buckminster fullerene consisting of sixty carbon atoms in a per- fect soccer ball structure. This solid is not stable at room temperature and exhibits totally different properties than diamond or graphite, even though all three materials consist exclusively of carbon atoms. Graphite is a semimetal, whereas diamond is a wide-band gap semiconductor with an energy gap of about $5.5 \mathrm{eV}$. Both semiconductors and insulator fullerenes can be doped to be metallic and even superconductors with transition temperatures exceeding $30 \mathrm{~K}$. Since 1985 when it was proposed to confine atoms in the cage structure of fullerenes, metal-containing fullerenes, have attracted a lot of interest in particular for technologically applications due to their combined fullerene-like and metallic properties [33]. Endohedral metallofullerenes (EMF) $\mathrm{MC}_{n}$ ( $\mathrm{M}=\mathrm{Sc}, \mathrm{Y}$, and the majority of the lanthanides) are carbon clusters with metal atoms encapsulated inside the cage $[34,35]$. The unique structure, physical and chemical properties of EMFs are of great interest as new materials with unique magnetic, electrical and optical properties [36, 37]. The production of EMFs is a real problem in studying the chemistry and physics of carbon clusters. Indeed, although the structures and electronic properties of many endohedral metallo-fullerenes have been extensively investigated, many of the properties of these compounds are still not well defined because of their limited availability. The maximum entropy method based on synchrotron X-ray powder diffraction Rietveld analysis has been used for structural determination of endohedral metallo-fullerenes such as $\mathrm{YC}_{82}, \mathrm{LaC}_{82}, \mathrm{La}_{2} \mathrm{C}_{80}$, $\mathrm{Sc}_{2} \mathrm{C}_{66}, \mathrm{Sc}_{2} \mathrm{C}_{2} \mathrm{C}_{84}, \mathrm{Sc}_{3} \mathrm{C}_{82}, \mathrm{GdC}_{82}, \mathrm{EuC}_{82}$ etc. From the MEM/Rietveld analysis of $\mathrm{YC}_{82}, \mathrm{LaC}_{82}$ and $\mathrm{ScC}_{82}$, Takata et al. suggested that the metal atom was located at an off-centered position on the $\mathrm{C}_{2}$ axis adjacent to a hexagonal ring of the $\mathrm{C}_{82}$ cage [38]. However, for both $\mathrm{EuC}_{82}$ and $\mathrm{GdC}_{82}$ an anomalous structure in which the metal atom is located on the $\mathrm{C}_{2}$ axis but adjacent to the $\mathrm{C}-\mathrm{C}$ double bond on the opposite side of the $\mathrm{C}_{2 \mathrm{v}}-$ $\mathrm{C}_{82}$ cage has also been reported in a clear disagreement with other experimental studies and theoretical calculations. In a recent work on the $\mathrm{GdC}_{82}$ system X-ray absorption near-edge structure (XANES) experiments have been able to identify the location of the Gd ion in the $\mathrm{GdC}_{82}$, an issue that has been a matter of debate with several possible models [39]. XANES data identified the Gd local structure inside the $\mathrm{C}_{82}$ fullerene cage as the $\mathrm{C}_{2}$-hexagon position characterized by a true minimum. Moreover, a temperature dependent dynamic behavior has been observed suggesting an oscillation of the metal ion on the flat potential energy surface with an amplitude increasing with temperature. Similar dynamic behavior has also been observed for the La ion in $\mathrm{LaC}_{82}$, with a large difference between the low and room temperature spectra, supporting the occurrence of a wide metal oscillation. The structural study of the $\mathrm{GdC}_{82}$ metallo-fullerene demonstrates the unique capabilities of the XANES technique for structural determinations of endohedral metallo-fullerenes. However, XANES spectroscopy alone is not able to identify and fully character- 
ize isomers, i.e., compounds with the same molecular formula but different structural formulae. There are many different classes of isomers that do not necessarily share similar properties. Actually, there are two main forms of isomerism: structural isomerism and stereoisomerism. In structural isomers, the atoms and functional groups are joined together in different ways. In stereoisomers the bond structure is the same, but the geometrical positioning of atoms and functional groups in space differs. While structural isomers are typically characterized by different chemical properties, stereoisomers behave identically in most chemical reactions. All fullerene cluster structures may be detected and characterized by their characteristic vibrational (breathing) modes [40]. Moreover, vibrational spectra are sufficiently sensitive to distinguish the various isomers. Nevertheless, comparison of IR experimental spectra may be not sufficient since the fullerene cage may have several isomeric forms with similar frequencies [41]. IR spectroscopy is a fundamental technique to recognize and investigate different endohedral metallo-fullerenes and their isomers and characterize the observed dynamic behavior of the metal inside the cage in particular if coupled with an X-ray spectroscopic method such as XANES, sensitive to the local structure and to the electronic properties, e.g., valence, spin, empty partial density of states, etc.

IR and X-ray studies are fundamental because fullerenes exhibit unique properties that render them suitable for many technological applications. As an example, they can be used in organic solar cells, transistors, and holographic materials. In particular, endohedral metal doped-fullerenes offer great opportunities in optoelectronic devices since varying the encapsulated metal cluster we may tune optical and electronic properties, without changing the structural features of the outer carbon shell.

Finally, similar to metallo-fullerenes we have to mention metalloproteins, a large set of proteins that includes up to about $30 \%$ of known proteins. Metalloproteins are a special class of proteins that combine the unique properties of metal atoms with the macromolecular assembly to perform specific biological processes. A large number of metalloproteins are also involved in many disease states including ageing processes. Although the mechanisms are not completely understood, we know that structural distortions of the metal site or inappropriate incorporation of metals may lead to characteristic diseases such as the amyotrophic lateral sclerosis (ALS) (or motor neuron disease) or the Creutzfeld-Jacob disease (CJD). In metalloproteins, the combined use of X-ray crystallography, XAS and FTIR spectroscopy is extremely useful. Indeed, the function(s) of many proteins is only partially understood and/or still unknown because the knowledge of a protein structure alone does not immediately clarify its molecular mechanism or its biological roles. Although proteins are typically characterized by more than 20000 vibrational degrees of freedom, e.g., the normal modes of vibration that may be correlated with internal coordinates such as bond lengths and bond angles, IR spectroscopy is a powerful technique sensitive to protein conformations and dynamics that can be performed in real physiological environments [42]. In fact, a protein spectrum is characterized by two main bands: the amide I and amide II that arise from specific stretching and bending vibrations of the peptide backbone. It is now well established that the band intensity and the frequency of the amide I band are sensitive to the protein secondary structure. By using infrared SR the secondary and tertiary structures of a protein can be resolved with extreme space and time resolutions and, with this technique, many relevant results have been achieved also in terms of chemical imaging of living cells and investigations of cellular processes. Regarding time resolution, analogous to metallo-proteins also metallo-enzymes play a prominent role in the metabolic pathways of all organisms. In addition, the catalytic reactions of metallo-enzymes can be described as a sequence during which different intermediate states occur which differ with respect to oxidation state and structure of the metal center. The static picture of a metal center as determined by crystallography, is not sufficient and the knowledge of the oxidation-state and of the structural changes at the metal center is really crucial for the understanding of time-resolved catalytic reactions of most metallo-enzymes. Moreover, the characterization of reaction intermediates with $\mathrm{X}$-ray spectroscopy coupled to vibrational data collected simultaneously on the same sample is a mandatory condition for the understanding of catalytic mechanisms. Synchrotron radiation sources have enough intensity to characterize the structure of reaction intermediates of metallo-enzymes. Rapid-scan XAS technique is a promising tool for studying state transitions at biological metal centers under physiological conditions. Using an accurate control of the monochromator (with piezoelectric devices) quick-EXAFS spectra can be obtained within $50 \mathrm{~ms}$ on relatively dilute samples ( $<10 \mathrm{mM}$ metal concentration) and in fluorescence mode $[43,44]$. Using the rapid-scan synchronous mode of both the monochromator and the undulator gap, at the beamline ID26 at the ESRF room-temperature EXAFS spectra of manganese complexes have been collected with not less than $3 \mathrm{~s}$ of scan time (for a $600 \mathrm{eV}$ scan) after the laser-flash excitation inducing a state transition [45-48]. For these studies, a novel fluorescencemode BioXAS technique has been also introduced. This sampling-XAS allows monitoring of state transitions and resolve intermediates with a time resolution at room temperature of non-crystalline metalloenzyme samples at (almost) physiological conditions from microsecond to second [49]. Similar time-resolved X-ray absorption spectroscopy experiments have been performed to the study of the active site zinc ion of bacterial alcohol dehydrogenase, a zinc-dependent enzymes that plays important roles in many cellular processes [50]. The assignment of the reaction mechanisms in many $\mathrm{Zn}$ enzymes is a matter of debate because zinc is silent in several spectroscopic 
techniques. In the work of Kleifeld et al. a series of alternations in the coordination number and structure of the catalytic zinc ion have been detected with concomitant changes in metal-ligand bond distances. The structural changes are correlated to the effective charge of the metal ion. These pioneering experiments clearly address the potential of time-resolved simultaneous studies to advance the understanding of the biological mechanisms occurring in the ms domain and, possibly, in the future thanks to the availability of ultrabright radiation sources in the $\mu$ s time domain. The understanding of the complex biological interactions does not require only time resolution but also new analytical techniques coupling a high spatial resolution and atomic and/or molecular sensitivity. As the final example of such systems we may address a recent X-ray absorption study of proteins adsorbed on the single-walled carbon nanotube (SWCNT) surface. XANES has been considered to investigate and characterize structural details of the protein-nanotube interface where small but relevant structural distortions occur. Specific XANES spectral features due to the combination of the protein hydrophobic pocket and of the SWNT hydrophobic surface, associated to the peptide $\mathrm{C}=\mathrm{O}$ bonds of the protein were recognized and found to be affected by the aromatic structure of SWCNTs [51]. This study confirms that XANES is really a powerful and almost unique tool capable of characterizing fine structural details of proteins at the interface of complex systems. Moreover, it demonstrates how the combination with IR vibrational spectroscopy may address additional details of the interaction occurring at the interface. The combination of IR and X-ray techniques could be also applied to the investigation of many different hybrid materials and to reconstruct complex interactions occurring at the interface of bio-nano systems.

At present, the majority of the concurrent/ simultaneous experiments outlined above, in particular those associated to nanosystems or to dynamical process characterized by fast timescale $(<1 \mathrm{~s})$ are mainly limited by source intensities. Actually, experiments combining a conventional IR spectrometer and an $\mathrm{X}$-ray synchrotron radiation source are limited in term of spatial and temporal resolution.

\section{Conceptual design}

The demand of simultaneous analytical experiments makes the question how to design and optimize instrumentation combining X-ray and IR beams for concurrent experiments. As addressed in the previous section and as demonstrated in the past, it is possible to combine an $\mathrm{X}$-ray synchrotron radiation source and an IR conventional source to perform concurrent experiments. However, the range of feasible experiments is significantly limited by the brilliance of conventional sources and/or by the flux when attempting to time-resolved experiments in the sub-second time domain. This aspect will be better discussed in the next section in order to justify the design of a conceptually new layout for concurrent IR and
X-ray experiments combining two synchrotron radiation sources.

In this section we will describe a schematic layout for a dedicated beamline capable to combine X-ray and IR to perform at the same sample position, in situ, simultaneous time-resolved experiments. For what concern the transport of both X-ray and IR beams the layout of the beamline is based on standard optical systems while experimental apparata, instrumentations and detectors technology will require a significant commitment and an important research and development (R\&D).

Actually, a dedicated beamline for simultaneous IR and X-ray spectroscopy is a unique system composed by two independent branches collecting the radiation from the same bending magnet. A homogeneous source such as bending magnet source is here considered for its homogeneous emission and stability compared to an insertion device. The two beams can be transported separately by two different optical systems and then merged inside the experimental end station where X-ray and IR beams converge in a paraxial geometry. To guarantee the ultimate time-resolution, e.g., from ms possibly down to the $\mu \mathrm{s}$ regime, an energy dispersive layout has to be considered for both systems. However, while different X-ray energy dispersive setups have been already implemented at several SR facilities, IR spectrometers are rare and both the time resolution and the wave number resolution desired will require a significant research and development effort coupled to the use of large and expensive IR array detectors. A detailed discussion of the optical layouts and of the instrumentation is discussed in a Ph.D. thesis [52] and will be the subject of a forthcoming publication.

\subsection{Source considerations}

If we consider the existing SR sources almost all of them run with the same electron energies $(\approx 2-3 \mathrm{GeV})$ and have similar performances in the IR domain. However, also the X-ray emissions from bending magnet are substantially comparable in the standard energy domain from a few $\mathrm{keV}$ up to $25-30 \mathrm{keV}$ with critical energies ranging from 8 to $10 \mathrm{keV}$. As a first step avoiding the possibility to collect the edge radiation generated by an electron beam in fringe fields of a bending magnet, both IR and X-ray beams can be easily extracted from the same bending magnet using two independent adjacent ports, separated by a suitable angle. From a homogeneous bending magnet source we may typically collect about $40 \mathrm{mrad}$ and about $2-3 \mathrm{mrad}$ in the horizontal plane for the IR and X-ray branches, respectively. Two optical systems can be then designed to achieve the characteristics summarized in Table for the two independent optical layouts.

\subsection{The energy dispersive $X$ branch}

The beamline has to be designed to perform timeresolved experiments on small samples (down to $\approx$ $0.3 \mathrm{~mm}$ of diameter) so that the X-ray branch is based on an energy dispersive layout with a long horizontally bent 
TABLE

Main parameters of the optical systems.

\begin{tabular}{l|l}
\hline \hline \multicolumn{1}{c}{ X-ray layout in the energy dispersive configuration } \\
\hline photon energy & $5-35 \mathrm{keV}(K$-edge from Ti to Xe $)$ \\
resolution & $E / E, 1 \times 10^{-4}$ or better \\
photon flux & $10^{11}-10^{12}$ photon $/ \mathrm{s}$ \\
Si(111) at $10 \mathrm{keV}$ & \\
beam size & $\approx 300 \mu \mathrm{m}(V) \times 300 \mu \mathrm{m}(H)$ \\
\hline
\end{tabular}

\begin{tabular}{l|ll}
\hline \multicolumn{3}{c}{ Infrared layout with a dispersive spectrometer } \\
\hline $\begin{array}{l}\text { photon energy } \\
\text { resolution }\end{array}$ & $\begin{array}{l}20,000-100 \mathrm{~cm}^{-1}(0.5-100 \mu \mathrm{m}) \\
0.1-16 \mathrm{~cm}^{-1}\end{array}$ \\
$\begin{array}{l}\text { photon flux at } \\
\text { sample } 5 \times 10^{13} \quad(\text { photon } / \mathrm{s} / 0.1 \% \quad \mathrm{bw}) \\
\text { beam size }\end{array}$ & $\begin{array}{l}\text { at } 1000 \mathrm{~cm}^{-1}(10 \mu \mathrm{m}) \\
\approx 300 \mu \mathrm{m}(V) \times 300 \mu \mathrm{m}(H)\end{array}$
\end{tabular}

crystal. In this layout the polychromatic beam could be focused at the sample position by a bent crystal and the spectrum detected by a position-sensitive detector located at a proper distance from the sample. This layout avoids mechanical movements taking benefit also of the high stability of the modern third generation SR sources. On modern light facilities, experiments could be easily performed with such geometry with a time resolution in the microsecond domain.

A pre-optic system has to be designed according to the characteristic of the specific source in order to match the size of the bent crystal. However, if we neglect the source size and the Darwin width, the photon range collected by a bent crystal depends only by geometrical parameters and the length of the crystal $(L)$ is only a function of the angular acceptance of the crystal $(\delta)$, the source-crystal distance $(p)$ and the incident angle (Bragg angle) $\theta_{0}$ :

$$
L=\frac{p \delta}{\sin \theta_{0}}
$$

actually, the effective crystal length will be limited also by the effective rocking curve of the bent crystal. Working with a tangential focusing geometry

$$
\frac{1}{p}+\frac{1}{q}=\frac{2}{R_{t} \sin \theta_{0}}
$$

the energy band pass $E$ for a cylindrically bent crystal with a focal length $f$ and a $2 \gamma$ divergence can be calculated [53] with the equation

$$
\Delta E=E_{0} \cot \theta_{0} 2 \gamma\left(\frac{r}{2 f}-1\right) .
$$

Within this framework, an energy band pass of $\approx 700 \mathrm{eV}$ at the Ti $K$-edge, large for a XANES analysis and useful for EXAFS, can be achieved. In a practical layout, to reduce the contribution of the natural vertical divergence before the crystal, a first cylindrical (or elliptical) mirror may be used to focus the radiation in the vertical, while a second flat mirror may readjust the beam parallel to the storage ring plane.

The bent crystal is the heart of the dispersive geometry for which the energy resolution is determined by the source size and the spatial resolution of the detector.
However, also the Darwin width and the aberrations induced by crystal bending properties and its figure affect the performances of the X-ray layout. All these characteristics can be simulated with ray tracing packages such as SHADOW-XOP [54].

\subsection{The IR branch}

The IR branch has to be designed to transmit the widest IR range from the far to the mid-IR focusing the beam at the same spot location of the X-ray beamline. The first section of the IR layout may use the standard optical system available to the majority of the IRSR beamline and based on a combination of flat and aspherical mirrors. In this layout we have four mirrors; the first plane mirror is used as the extraction optic is followed by an ellipsoidal mirror focusing the light to a thin wedged diamond window. After the aspherical mirror, two cylindrical (or parabolic) mirrors can be used to collimate the beam into the two orthogonal planes. In the second section a set of mirrors will deflect and condense the IR radiation, as a function of the X-ray energy, inside a special optics where IR radiation will be focused to the sample position along a path paraxial to the X-ray beam (see Fig. 5). If we consider a IRSR source at $10 \mu \mathrm{m}$, an initial flux of $5 \times 10^{12}$ phot./s $/ 0.1 \%$ bw and the ab-

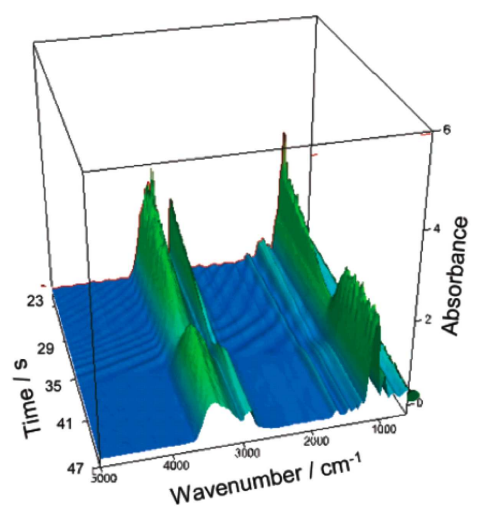

Fig. 4. Three-dimensional FTIR images obtained during the deposition by casting of a self-assembling silica film. The spectra have been recorded by rapid scan time-resolved method. In this plot when the fringes suddenly disappear we may clearly recognize the liquidsolid transition.

sorption of the diamond window, ray tracing calculations show that losses can be significantly reduced by a proper choice of both mirror size and figures. Actually, the main losses are determined by the aperture and by the central obscuration of the optical concentrator. Moreover, a reasonable estimation of the flux on the sample position is in the $10^{12}$ phot./s range at the wavelength of $10 \mu \mathrm{m}$ (with a spot size of $\approx 0.1 \mu \mathrm{m}^{2}$ ).

\subsection{The experimental station and the advantages of $S R$}

The foreseen combined use of X-ray and IR radiation in the framework of time-resolved experiments in the subsecond time domain down to a few microseconds imposes 


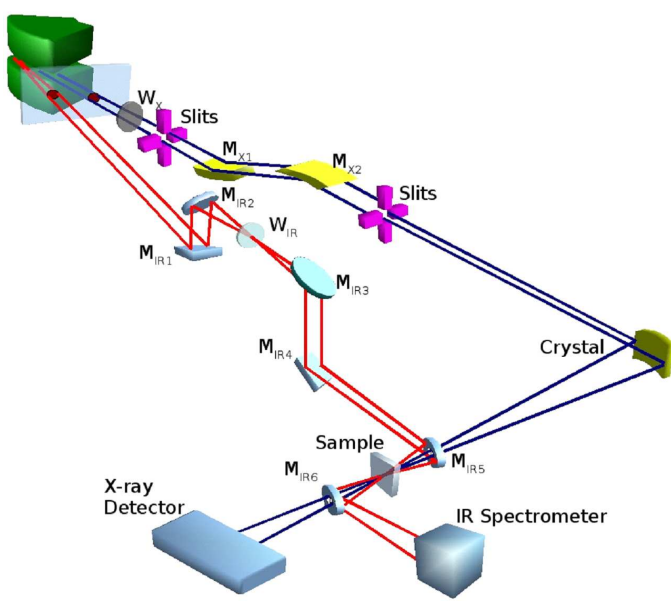

Fig. 5. Conceptual design of the IXSS beamline with the two independent optical systems. The X-ray system has a bent crystal while the IR beamline is equipped by an IR spectrometer. In the sample compartment the two beams are paraxial.

significant constraints to the design of all optical elements. In particular, based on the experience collected in many years at different SR facilities, in particular in the IR domain, where source instabilities are the main sources of noise, a great attention has to be devoted to all sources of noise - mechanical, electrical, thermal, and optical [55]. However, although present achievements on electron beam instabilities are encouraging, additional improvements are really welcome for future X-ray and IR simultaneous experiments [56]. A key issue of the experimental station will be the spectrometers set at the focal point. Indeed, the experimental station has to be designed for standard time resolved experiments in the transmission geometry. However, the spectrometer design is to be as user friendly as possible, with clear in mind the demand of flexibility and of versatility, necessary to carry out different types of experiments on different kind of samples. To this purpose the sample compartment will be integrated in a conceptually new spectrometer working in vacuum, with a sample stage common to both the X-ray and IR beams and sharing the same sample position of the spectrometer. Evaluating the performances of such beamline on a third generation synchrotron radiation is not an easy task without a precise knowledge of the source characteristics. At present, we may postpone an accurate calculation of the IR emission because in the IR domain we are limited by the instrument performances. Actually, in the rapid scan mode the faster interferometer may collect data down to a few ms. On the contrary, a rough evaluation of the time resolution of concurrent or simultaneous experiments for the $\mathrm{X}$-ray dispersive layout is possible considering the brilliance at the sample position, i.e., the integrated flux/s within one sterad solid angle on a unit sample area and the acquisition time, i.e., the time required to collect a transmission spectrum of a sample with the necessary
$\mathrm{S} / \mathrm{N}$ ratio. In the following evaluation of the performance of a time-resolved X-ray absorption experiment, we will start considering working at the iron $K$ edge $(7112 \mathrm{eV})$ over a sample area of about $250 \mu \mathrm{m}(H) \times 250 \mu \mathrm{m}(V)$. If we consider a minimum $\mathrm{S} / \mathrm{N}$, we may estimate the time necessary to collect a XAS spectrum in the energy dispersive geometry (from $\approx 100 \mathrm{eV}$ below the edge up to 800-1000 eV above the edge). In the transmission mode, spectra are collected using an ionization chamber set before the sample and a linear array. The latter could be the XH Ge Strip detector the world first $50 \mu \mathrm{m}$ pitch strip detector specifically designed for energy dispersive experiments with integration times in the range from $1 \mu \mathrm{s}$ to $1 \mathrm{~s}[57]$. We assume that $\beta$ is the fraction of $I_{0}$ (intensity before the first ionization chamber) absorbed by the first ionization chamber. After a sample of thickness $d$, the intensity will decrease and the transmitted intensity is

$$
I_{t}=I_{0}(1-\beta) \mathrm{e}^{-\mu_{t} d},
$$

where $\mu_{t}$ is the absorption coefficient of the sample. If all transmitted photons are detected by the linear array, the measured value is given the by ratio

$$
r=I_{0} \beta / I_{t}=\beta /\left[(1-\beta) \mathrm{e}^{-\mu_{t} d}\right],
$$

where we have to maximize the experimental accuracy when measuring changes in $r$ induced by small changes of $\mu$. Because

$$
S=\mathrm{d} r / \mathrm{d} \mu_{t}=d \beta /\left[(1-\beta) \mathrm{e}^{-\mu_{t} d}\right],
$$

if we consider the statistical noise of a measurement as

$$
\mathrm{S} / \mathrm{N}=d \sqrt{I_{0} /\left[1 / \beta+\mathrm{e}^{-\mu_{t} d} /(1-\beta)\right]},
$$

we may achieve the maximum $\mathrm{S} / \mathrm{N}$ ratio when $\beta=20 \%$ and $\mu_{t} d \approx 2.6$. However, a typical edge jump $\mu_{t} d$ ranges from 0.8 to 1.5 accompanied by a $20 \%$ photon absorption in the first ionization chamber [58]. Nevertheless, with an edge jump of 1.2 we may still collect reasonably good spectra in a wide energy range around the Fe $K$-edge, whose optimum absorption thickness is $5.1 \mu \mathrm{m}$ with a jump of 1.2 [59]. To measure Fe $K$ edge EXAFS oscillations whose typical signal ranges between $1 \%$ and $10 \%$ of the total absorption cross-section we need a noise significantly lower than $1 \%$, e.g., a $\mathrm{S} / \mathrm{N}>150$ [60]. As a consequence, from Eq. (6) we may obtain that the minimum intensity required for a transmission EXAFS measurement is $8 \times 10^{9}$ phot./s/0.1\% bw, and for the X-ray branch of the beamline, the time resolution for a EXAFS spectrum at the Fe $K$-edge up to $k \approx 15 \mathrm{~A}^{-1}$ is $\approx 240 \mathrm{~ms}$. The results is really encouraging because with the flux already available at third generation facilities $\left(\approx 10^{11}-10^{12}\right.$ phot./s $/ 0.1 \%$ bw $)$ time resolution of few ms are possible and the final goal of energy dispersive experimental acquisition in the microsecond domain appear feasible [61].

\section{Conclusions}

Synchrotron radiation and different spectroscopic techniques may be, respectively, the intense source and the 
reliable methods, capable to face up the demand of new experimental setup based on concurrent, possibly simultaneous, time-resolved analytical techniques. The existing convergence among materials science, biology and medicine that is merging together wide scientific communities requires a new vision and different analytical capabilities. Actually, the combination of two or more experimental techniques is a clear trend in several scientific areas and especially in frontier researches and crosscut research areas. To this purpose it is really remarkable to notice that only after about 10 years the same experiments performed at Daresbury [2] with a slightly different setup but combined to a more powerful synchrotron radiation source may offer such significant advantages [3]. Different combinations of techniques based on conventional IR or laser sources have been performed or are available routinely at different European synchrotron radiation facilities such as ANKA, APS, Bessy, Diamond, Elettra, ESRF, Soleil, SLS and SSRL. Experimental results have been already presented and discussed in conferences and workshops. At the last meeting held in Grenoble and organized by the Swiss-Norwegian beamline at ESFR [62] on June 2008 scientists from all over the world discussed about the results obtained combining different microscopy techniques and the future possibilities. The workshop demonstrates that imaging is a mature research field and the convergence of simultaneous and time-resolved techniques is an emerging analytical approach. Many applications of in situ time resolved combination of techniques are foreseen and we expect cooperation in many areas ranging from materials science, catalysis, soft- and nanochemistry, biology, nanotechnology, etc. The combination of mid-IR and X-ray simultaneous spectroscopy appears an optimal combination because they probe not only comparable voxels, but because it combines structural and electronic analysis at atomic resolution with a molecular analysis. The order reconstruction in space and time is a hallmark of many phenomena and the concurrent investigations with IR and X-ray allow the study of both (complex) materials and (dynamic) phenomena, e.g., the study of biological functions monitoring at the same time and vs. time and/or the dose the sample damage. As a consequence, the simultaneous combination of atomic or structural analysis with molecular analysis will offer immense rewards to materials science and will certainly improve the understanding of many fundamental processes.

From the experimental point of view the strategy of a concurrent analysis remains challenging. However, brilliant synchrotron sources may fulfill the experimental demands and indeed, although many researches could take benefit only performing a simple concurrent analysis in which the IR source is a conventional source, new research opportunities can be open using IR synchrotron radiation and new spectrometers in particular to exploit fast phenomena in the sub-second time domain.

\section{Acknowledgments}

We gratefully acknowledge the support of the Italian Ministry Foreign Affairs in the framework of the 12th Executive Programme of Scientific and Technological Cooperation between the Italian Republic and the People's Republic of China. In particular one of us (Wei Xu) gratefully acknowledges the support of the Italian Ministry Foreign Affair for financial support. We acknowledge also P. Morini and D. Sali of Bruker Optics for many fruitful discussions.

\section{References}

[1] P. Innocenzi, L. Malfatti, T. Kidchob, S. Costacurta, P. Falcaro, M. Piccinini, A. Marcelli, P. Morini, D. Sali, H. Amenitsch, J. Phys. Chem. C 111, 5345 (2007).

[2] W. Bras, G.E. Derbyshire, D. Bogg, J. Cooke, M.J. Elwell, B.U. Komanschek, S. Naylor, A.J. Ryan, Science 267, 996 (1995).

[3] M.A. Newton, A.J. Dent, S.G. Fiddy, B. Jyotid, J. Evans, Phys. Chem. Chem. Phys. 9, 246 (2007).

[4] R.J. Davies, M. Burghammer, C. Riekel, Macromolecules 39, 4834 (2006).

[5] M.A. Newton, A.J. Dent, S.G. Fiddy, B. Jyoti, J. Evans, Catalysis Today 126, 64 (2007).

[6] B.S. Clausen, H. Topsøe, R. Frahm, Adv. Catal. 42, 315 (1998).

[7] G.S. Sankar, J.M. Thomas, Top. Catal. 8, 1 (1999).

[8] J.G. Mesu, A.M.J. van der Eerden, F.M.F. de Groot, B.M. Weckhuysen, J. Phys. Chem. B 109, 4042 (2005).

[9] S. Diaz-Moreno, D.T. Bowron, J. Evans, Dalton Trans. 23, 3814 (2005).

[10] G. Guilera, M.A. Newton, C. Polli, S. Pascarelli, M. Guino, K.K. Hii, Chem. Commun. 41, 4306 (2006).

[11] A.M. Beale, A.M.J. van der Eerden, K. Kervinen, M.A. Newton, B.M. Weckhuysen, Chem. Commun. 24, 3015 (2005).

[12] V. Briois, D. Lutzenkirchen-Hecht, F. Villain, E. Fonda, S. Belin, B. Griesebock, R. Frahm, J. Phys. Chem. A 109, 320 (2005).

[13] J.D. Grunwald, M. Ramin, M. Rohr, A. Michailovski, G.R. Patzke, A. Baiker, Rev. Sci. Instrum. B 76, 054104 (2005).

[14] X-ray Absorption: Principles, Applications, Techniques of EXAFS, SEXAFS, XANES, Eds. D.C. Konigsberger, R. Prins, Wiley, New York 1988.

[15] R. Phillips, S.R. Quake, Phys. Today 59, 38 (2006).

[16] N.A. Young, J. Chem. Soc., Dalton Trans. , 249 (1996).

[17] O.M. Wilkin, N.A. Young, J. Synchrotron Rad. 6, 204 (1999).

[18] M. Cestelli Guidi, M. Piccinini, A. Marcelli, A Nucara, P. Calvani, E. Burattini, J. Opt. Soc. Am. A 22, 2810 (2005).

[19] C.J. Brinker, Y. Lu, A. Sellinger, H. Fan, Adv. Mater. 11, 579 (1999). 
[20] P. Innocenzi, T. Kidchob, L. Malfatti, S. Costacurta, M. Takahashi, M. Piccinini, A. Marcelli, J. Sol.-Gel. Sci. Technol. 48, 258 (2008).

[21] G.D. Smith, R.A. Palmer, in: Handbook of Vibrational Spectroscopy, Eds. J.M. Chalmers, P.R. Griffiths, Wiley, Chichester 2002, Vol. 1, p. 626.

[22] P. Innocenzi, T. Kidchob, J. Mio Bertolo, M. Piccinini, M. Cestelli Guidi, A. Marcelli, J. Phys. Chem. $B$ 110, 10837 (2006).

[23] M. Antoietti, G.A. Ozin, Chem. Eur. J. 10, 28 (2004).

[24] C.J. Brinker, D.R. Dunphy, Curr. Opin. Coll. Inter. Sci. 11, 126 (2006).

[25] M. Takahashi, T. Maeda, K. Uemura, J. Yao, Y. Tokuda, Y. Yoko, Y. Kaji, A. Marcelli, P. Innocenzi, Adv. Mater. 19, 4343 (2007).

[26] R. Peierls, Proc. Phys. Soc. London, Ser. A 49, 72 (1937).

[27] M. Imada, A. Fujimori, Y. Tokura, Rev. Mod. Phys. 70, 1039 (1998).

[28] A. Sacchetti, M. Cestelli Guidi, E. Arcangeletti, A. Nucara, P. Calvani, M. Piccinini, A. Marcelli, P. Postorino, Phys. Rev. Lett. 96, 035503 (2006).

[29] S.-W. Cheong, H.Y. Hwang, in: Colossal Magnetoresistance Oxides, Ed. Y. Tokura, Monographs in Condensed Matter Science, Gordon and Breach, Reading, U.K. 2000, p. 237.

[30] A.J. Millis, B.I. Shraiman, R. Mueller, Phys. Rev. Lett. 77, 175 (1996)

[31] A.J. Millis, Nature 392, 147 (1998).

[32] W. Eberhardt, Surf. Sci. 500, 242 (2002).

[33] H.W. Kroto, J.R. Heath, S.C. O'Brien, R.F. Curl, R.E. Smalley, Nature 318, 162 (1985).

[34] J.R. Heath, S.C. O'Brien, Q. Zhang, Y. Lui, R.F. Curl, H.W. Kroto, R.E. Smalley, J. Am. Chem. Soc. 107, 7779 (1985).

[35] D.S. Bethune, R.D. Johnson, J.R. Salem, M.S. de Veles, C.S. Yannoni, Nature 336, 123 (1993).

[36] H. Shinohara, Rep. Prog. Phys. 63, 843 (2000).

[37] J.R. Heflin, D. Marciu, C. Figura, S. Wang, P. Burbank, S. Stevenson, H.C. Dorn, Appl. Phys. Lett. 72 , 2788 (1998).

[38] M. Takata, E. Nishibori, M. Sakata, H. Shinohara, Struct. Bonding 109, 59 (2004).

[39] L. Liu, B. Gao, W. Chu, D. Chen, T. Hu, C. Wang, L. Dunsch, A. Marcelli, Y. Luo, Z. Wu, Chem. Commun. 4, 474 (2008).

[40] M.S. Dresselhaus, G. Dresselhaus, P.C. Eklund, Science of Fullerenes and Carbon Nanotubes, Academic Press, San Diego 1996.

[41] S. Guha, K. Nakamoto, Coord. Chem. Rev. 249, 1111 (2005).

[42] A. Barth, C. Zscherp, Quart. Rev. Biophys. 35, 369 (2002).

[43] D. Lützenkirchen-Hecht, S. Grundmann, R. Frahm, J. Synchrotron Rad. 8, 6 (2001).
[44] M. Richwin, R. Zaeper, D. Lützenkirchen-Hecht, R. Frahm, Rev. Sci. Instrum. 73, 1668 (2002).

[45] M. Haumann, M. Grabolle, M. Werthammer, L. Iuzzolino, J. Dittmer, W. Meyer-Klaucke, T. Neisius, H. Dau, PS2001 Proceedings, Contribution S10-013, p. 1 (2001).

[46] M. Haumann, M. Grabolle, T. Neisius, H. Dau, FEBS Lett. 512, 116 (2002).

[47] M. Haumann, P. Pospisil, M. Grabolle, C. Muéller, P. Liebisch, V.A. Sole', T. Neisius, J. Dittmer, H. Dau, J. Synchrotron Rad. 9, 304 (2002).

[48] H. Dau, M. Haumann, J. Synchrotron Rad. 10, 76 (2003).

[49] M. Haumann, C. Muller, P. Liebisch, T. Neisius, H. Dau, J. Synchrotron Rad. 12, 35 (2005).

[50] O. Kleifeld, A. Frenkel, J.M.L. Martin, I. Sagi, Nature Struct. Biol. 10, 98 (2003).

[51] J. Zhong, L. Song, J. Meng, B. Gao, W. Chu, H. Xu, Y. Luo, J. Guo, A. Marcelli, S. Xie, Z. Wu, Carbon 47, 967 (2009).

[52] Xu Wei, Ph.D. Thesis, Graduate school of Chinese Academy of Sciences, Institute of High Energy Physics 2008.

[53] F. D'Acapito, F. Boscherini, A. Marcelli, S. Mobilio, Rev. Sci. Instrum. 63, 899 (1991).

[54] M. Sánchez del Río, R.J. Dejus, in: Synchrotron Radiation Instrumentation: Eighth Int. Conf., Eds. T. Warwick, J. Arthur, H.A. Padmore, J. Stöhr, American Institute of Physics, 2004, p. 784.

[55] R.O. Hettel, Rev. Sci. Instrum. 73, 1396 (2002).

[56] M.F. Brigatti, D. Malferrari, M. Poppi, A. Mottana, G. Cibin, A. Marcelli, G. Cinque, Am. Mineral. 93, 821 (2008).

[57] J. Headspith, J. Groves, P.N. Luke, M. Kogimtzis, G. Salvini, S.L. Thomas, R.C. Farrow, J. Evans, T. Rayment, J.S. Lee, W.D. Goward, M. Amman, O. Mathon, S. Diaz-Moreno, in: IEEE Nuclear Science Symp. Conf., Ed. M.W.T. Benjamin, IEEExplore 4, 2007 , p. 2421.

[58] G.A. De Rose, H. Jiang, P.D. Holland, W.W. Adams, R.W. Hoffman, C.A. Zorman, Inter. Rep. Air Force Material Command (Ohio) Jan.-Sep. 1993, WL-TR93-4107, p. 11.

[59] http://cars9.uchicago.edu/ newville/mcbook/.

[60] J.B. Hastings, in: Applications of EXAFS to Materials Science Conf., BNL-28946, http://www.osti.gov/ bridge/product.biblio.jsp?osti_id $=6862052$.

[61] S. Pascarelli, O. Mathon, M. Munoz, T. Mairs, J. Susini, J. Synchrotron. Rad. 13, 351 (2006).

[62] Workshop on Simultaneous Raman-X-ray Diffraction/Absorption Studies for the In Situ Investigation of Solid State Transformations and Reactions at Non Ambient Conditions, Grenoble 2008, at: http://www.esrf.eu/UsersAndScience/Experiments/ CRG/BM01/raman_workshop/report. 